(C) 2017 IEEE. Personal use of this material is permitted. Permission from IEEE must be obtained for all other uses, in any current or future media, including reprinting/republishing this material for advertising or promotional purposes, creating new collective works, for resale or redistribution to servers or lists, or reuse of any copyrighted component of this work in other works. 


\title{
Design of Contour based Protection Zones for Sublicensing in Spectrum Access Systems
}

\author{
Huiyang Wang*, Eryk Dutkiewicz*, Beeshanga Abewardana Jayawickrama*, Markus Dominik Mueck ${ }^{\dagger}$ \\ ${ }^{*}$ School of Computing and Communications, University of Technology Sydney, Australia. \\ $\dagger$ Intel Mobile Communications, Germany. \\ Email: huiyang.wang@student.uts.edu.au, \\ \{eryk.dutkiewicz, beeshanga.jayawickrama\}@uts.edu.au, markus.dominik.mueck@intel.com
}

\begin{abstract}
Spectrum Access System (SAS) allows incumbent military systems to share spectrum in a hierarchical manner with tier-2 Priority Access License (PAL) users and tier-3 General Authorized Access (GAA) users. FCC has recently allowed PAL owners to sublicense their channels. Therefore, when GAA channels are congested they can request a sublicense to access the PAL channel on a coordinated basis, which provides interference protection from other GAA users. In this paper we propose a grid map to measure and monitor the secondary spectrum market for the purpose of spectrum trading with QoS guarantee. This work provides the subsequent spectrum trading models with a reasonable and dedicated interference graph for further optimization of spectrum allocation. Compared with traditional longterm spectrum licensing policy, short-term licensing makes the spectrum allocated effectively. We find the optimal resolution of the discrete grid map that maximizes the profit from sublicensing. Simulation results are provided to demonstrate how fine to grid the region and let the PAL owner achieve monetary benefit, in a given number of sensors.
\end{abstract}

\section{INTRODUCTION}

With the rapid development of mobile networks, a nationwide spectrum license authorized for a long term is no longer efficient. In the coming $5 \mathrm{G}$ era, a short-term license affords more flexibility and efficiency for both wireless users and providers. As a novel economic resource, spectrum should be dynamically managed in the further networks both in theory and practice. How to design the policy of access becomes a critical problem. Exclusive spectrum access and unlicensed access are two basic methods. Exclusive spectrum access will certainly be needed in $5 \mathrm{G}$ mobile systems to guarantee quality of service (QoS) while unlicensed access, on the contrary, offers unpredictable QoS but for free. However, other regulatory options may be needed in addition to coordinate the interference between them. For example, in 2011 the Radio Spectrum Policy Group (RSPG) in Europe proposed Licensed Shared Access (LSA) for spectrum sharing [1] [2], and in 2012 USA's Federal Communications Commission (FCC) started to develop Spectrum Access System (SAS) [3] [4].

Considering interference issue, the cell planning is a crucial part of base station deployment within one single operator's communication network. Although inter-operators' sharing can improve the spectrum efficiency and many algorithms are proposed for this purpose, the problem has been remained challenging in multiple operators because of private issues and mutual interference [5] [6]. Fortunately, SAS can provide a
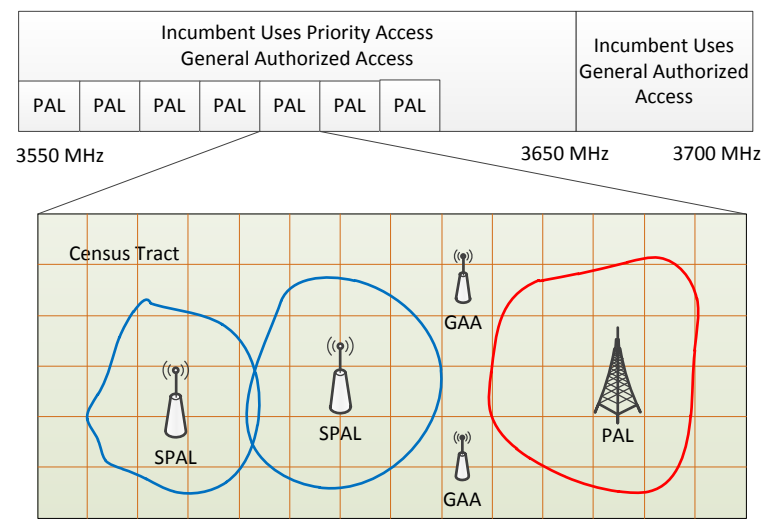

Fig. 1. SAS: A Priority Access License (PAL) is defined as a non-renewable authorization to use a $10 \mathrm{MHz}$ channel in a single census tract for three years The PAL holder could be any kinds of stakeholders, including who even do not have infrastructures and they can lease the spectrum to a Secondary PAL owners. The protection zones can be defined in a dedicated grid map.

flexible platform for multi-operators' spectrum sharing. It is a three-tiered framework, including (i) Incumbent Access (highest privilege), (ii) Priority Access and (iii) General Authorized Access (GAA) (lowest privilege).

In this paper, we focus on the spectrum spatial efficiency of Priority Access License (PAL) in SAS. The latest FCC report [4] proposed to allocate the PAL by auction for threeyear term on census tract basis. The Priority Access Licensees could be anyone, even those who have no infrastructure. The PAL owner can use the spectrum with protection from the GAA users' interference. In some cases, the PAL owner can lease a sublicense to a Secondary PAL (SPAL) user for the purpose of monetary. As a charged user, the SPAL user also needs protection from harmful interference from other SPAL and GAA users. One of the challenging problems is how to define the protection zone for each owner and SPAL user. Although the protection zone is defined as $-96 \mathrm{dBm}$ contour in [4], the coordination between different stakeholders is not given. To ensure exclusive sublicenses we introduce a spatial interference-free grid map to formalize and minimize the interference, as shown in Fig. 1.

The spectrum allocation between multiple operators is investigated in [7] and spectrum sharing in heterogeneous network is analyzed in [8] [9]. To solve the competition, 
economic tool is also widely used to solve the conflicting problem in a dense region [10] [11]. In a business driven model, SPAL users pay PAL owner for the sublicenses, in return, PAL owner needs to provide them with QoS guarantee. However, none of the existing works mention how to construct the interference restraints based on the protection zone. To our best knowledge, this is the first paper present detailed solution of protection zones.

The novelty of this paper is adopting grid to solve complex interference contours among multiple operators. By taking the advantage of the fact that the power falls off with distance of signal propagation, we can reuse the same frequency at spatially separated locations. For a given region, it can be easily divided into cells by one operator, so long as the interference between co-channels stations is kept below acceptable levels. When it comes to sharing of multiple operators, the easy way to coordinate interference is pre-defining protection zones, which involves few steps computation. In our method, each active $-96 \mathrm{dBm}$ contour represents a base station's coverage area. The grid criteria includes the a proper resolution and maximizing the profit of spectrum trading. Simulation results show how fine to grid a region and achieve a higher profit.

The rest of the paper is organized as follows. In Section II, the preliminaries and problem formulation are provided. In Section III, we present the details of our proposed solution. We also provide simulation result and discussion in Section IV, and conclude the paper in Section V.

\section{Preliminaries And Network Model}

\section{A. Preliminaries}

Inter-operator shared access is a complementary new alternative for spectrum sharing, where multiple operators jointly use a part of licensed spectrum. Even though it can improve the spectrum efficiency, the exclusive use and interference protection from each other turn out to be new challenges. SAS can enable the short-term licenses but still need to call for more flexible regulatory regime where, for example, different operators may use exclusive bands with different authorization modes, such as PAL and GAA. PAL is a license lasting for three years in a census tract [4] with interference protection, while GAA is based on opportunistic access and without any QoS guarantee. When the GAA band is congested, more operators will seek good QoS from PAL band. In this case, PAL owner can earn money by leasing a sublicense.

The SAS will incorporate information from a sensing network, which will be used to detect available spectrum and monitor the behavior of SPAL users, e.g. SPAL users' actual contours. Moreover, given the critical importance of the interference-free operations in the band, we must ensure that SPAL users' base stations submit their contours as their protection zones and prices. Then SPAL users can be protected in the same privilege as the PAL users.

Both PAL and SPAL users should report their protection contours on the basis of the network deployment, as long as they are within the boundaries established by the objective default protection contour. They are encouraged to work with
SAS Administrators to tailor their self-reported PAL protection zone to their particular needs within the boundaries defined by the default protection contours. The approach will provide flexibility to PAL user while also creating an objective means of determining a maximum protection contour and minimizing the risk that PAL user might claim protections beyond the extent of their actual network deployment. Under a system relying on pure self-reporting, we are concerned that PAL user would be effectively encouraged to deploy their networks inefficiently and seek protection for extremely low signal levels or in areas without facilities that are in actual use.

\section{B. Network Model}

The spectrum sharing framework can be illustrated with a set of $N$ base stations competing for limited number of sublicenses from a PAL user. Every potential base station needs to submit its $-96 \mathrm{dBm}$ contour, which is used to win the sublicenses in the space domain, then the SAS sever will compute the interference-free protection zones and then allocate sublicenses to them. The shape of the contour actually could be arbitrary and it is difficult to tell the interference condition between them in a distributed regime. Therefore the centralized SAS is more suitable for the sublicensing allocation. Let the shape of contour $i$ be $D_{i}: F(x, y)=0$, and its payment is

$$
p_{i}=\iint_{D_{i}} v_{i}(x, y) d \sigma
$$

where $v_{i}(x, y)$ is the valuation density in the space domain. The spectrum allocation should ensure that contours are spatially independent, namely the protection zone could not overlap with any other one. The allocation result is denoted by $a_{i} \in\{0,1\}$. If SPAL user $i$ is assigned a sublicense $a_{i}=1$, otherwise $a_{i}=0$ and accordingly it pays nothing, $p_{i}=0$. PAL user's revenue $\mathcal{R}$ of the sublicensing comes from all the SPAL users, which defined as

$$
\mathcal{R}=\sum_{i=1}^{N} a_{i} \iint_{D_{i}} v_{i}(x, y) d \sigma
$$

The cost of the PAL user is the number of sensors it uses to monitor the interference in the census tract. Assume that the density of sensors over the census tract is $\rho(x, y)$, which is the number of sensors over per unit space. Let $\eta$ denote the cost of one sensor in term of monetary $\$$. Therefore, the cost of sensing network over the area $D_{i}$ is

$$
c_{i}=\eta \iint_{D_{i}} \rho(x, y) d \sigma
$$

The objective is to maximize the profit of sublicense trading, defined as

$$
\begin{aligned}
\mathcal{P}(\mathbf{D}, \mathbf{v}, \rho(x, y)) & =\arg \max _{a_{i}} \mathcal{R}-\sum_{i=1}^{N} a_{i} c_{i} \\
& =\arg \max _{a_{i}} \sum_{i=1}^{N} a_{i} \iint_{D_{i}} v_{i}(x, y)-\eta \rho(x, y) d \sigma
\end{aligned}
$$




$$
\text { s.t. } D_{i} \cap D_{j}=\varnothing, \forall i, j
$$

where $\mathbf{D}$ is the set of submitted contour areas $\mathbf{D}=$ $\left\{D_{1}, D_{2}, \ldots, D_{N}\right\}$ and $\mathbf{v}=\left\{v_{1}, v_{2}, \ldots, v_{N}\right\}$. The constraint (5) aims to guarantee there is no overlapping between any two SPAL users' contours.

\section{Protection Zone In GRID}

The traditional homogeneous network expansion technologies will no longer handle the rapid growth of user population and their wireless volume thirsty [12]. In the future the heterogeneity of network will become more prominent, in terms of cell size and access methods. Moreover, the shape and size of potential SPAL users heavily differ from each other. One of the feasible way is to define a protection zone for the sublicensees. Spatial division is more flexible and with low computation. A design of sublicenses over grid is demonstrated in this section.

\section{A. Grid Resolution}

The shape of contour is arbitrary because of geographical environment, which makes the protection zone difficult to measure in practice. The cost of sublicense is based on the area of the contour then the immediate question is how to estimate it.

The common method to estimate the area of an arbitrary shape is breaking it up into smaller basic shapes, which will help approach to a fine approximation. The smaller the shapes are, the better the approximation will be. One easy way is putting the arbitrary shape into a grid and count the number of tiles it covers. We assume that the sensors are uniformly deployed in a region, and at the nodes in a grid, as shown in Fig. 2. The resolution of a grid is

$$
\rho(x, y)=\left(\frac{1}{\lambda}+1\right)^{2} \approx \frac{1}{\lambda^{2}}, \lambda \ll 1
$$

where $\lambda$ is the size of the tile or the distance between two sensors in the same row/column, e.g. $\lambda_{a}=1 / 6 \mathrm{~km}$ in Fig. 2 (a) and $\lambda_{b}=1 / 8 \mathrm{~km}$ in Fig. 2 (b).

Then we use two types of tiles to estimate the area of the contour: boundary/half tile and interior/whole tile. Because partial covering of a tile is usually count as a half tile. The boundary is covered by half tiles and the interior is covered by whole tiles. In fact, the boundary area, which is covered by partial tiles could also be another base station's boundary, so another parameter $\beta$ is defined as the boundary sensitivity and it will be explained in Section B III. The price of the contour $i$ is estimated as the unit space valuation multiplied by the contour area

$$
p_{i} \approx v_{i}\left(w_{i}+0.5 \beta h_{i}\right) \lambda^{2}
$$

where $w_{i}$ is the number of whole tiles in a contour $i$, and $h_{i}$ is the number of half tiles in a contour, and $v_{i}$ is the average valuation per unit space for the sublicense. The coefficient 0.5 is used to estimate area of the half tile as 0.5 , i.e., one half of the whole tile.

For example, in the grid of Fig. 2(a) the number of whole tile is $w_{a}=9$, while the number of half tiles is $h_{a}=20$.

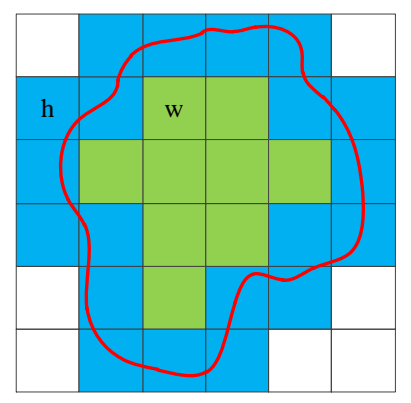

(a)

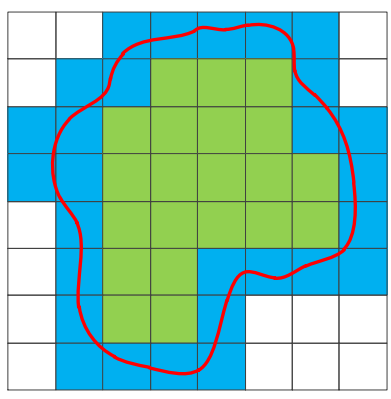

(b)
Fig. 2. The grid is used to measure and monitor the protection zone. In this example, the area is normalized as $1 \mathrm{~km} \times 1 \mathrm{~km}$. The resolution in (a) is lower than that in (b), i.e., $\lambda_{a}=1 / 6 \mathrm{~km}, \lambda_{b}=1 / 8 \mathrm{~km}$.

In Fig. 2(b), the number of whole tile is $w_{b}=21$ while the number of half tiles $w_{b}=26$. Therefore the prices under two different resolutions are

$$
\begin{aligned}
& p_{a}=v\left(w_{a}+\beta h_{a}\right) \lambda_{a}^{2}=v(9+20 * 0.5 \beta) / 6^{2} \\
& p_{b}=v\left(w_{b}+\beta h_{i}\right) \lambda_{b}^{2}=v(21+26 * 0.5 \beta) / 8^{2}
\end{aligned}
$$

respectively.

Note that though a higher resolution contributes to a good precision, but it needs a larger number of sensors and also results in higher cost. To guarantee interference protection between SPAL users, sublicenses should be assigned to nonoverlapping contours, the allocation determination should optimize the profit. Potential SPAL users apply to the SAS sever for temporary sublicensing protection. Success of the competing for protection depends on their pricing and overall determination in a conflicting situation. The sublicense rights are granted by the overall judgment.

\section{B. Boundary Sensitivity}

In a dense area, where a large number of SPAL users compete for sublicenses, the interference on the boundary area is difficult to tell from each other. Because in some cases, the boundary tile is usually shared by more than one SPAL user. This is the reason we introduce boundary sensitivity $\beta$, which is defined as the tolerance of the neighbors. It is used to judge the maximum number of shared users in a boundary tile. For example, if the boundary tiles are not allowed to share with anyone else, the boundary sensitivity $\beta$ is set as zero. In a sparse situation, the boundary area is still interference-free, and boundary sensitivity $\beta$ should be set as zero, which means that the sublicensee does not want to share the boundary tiles with anyone else. On the contrary, in a competitive situation, the boundary tile is probably shared by multiple base stations. Since the QoS is no longer as good as that in a sparse situation, the boundary is less valuable. In this case, the boundary sensitivity is set as the tolerance in terms of the limited number of SPAL users over the boundary area. If the number of shared users in a boundary tiles is greater than the boundary sensitivity, the tiles will not be charged and no QoS guarantee. 
For example, in Fig. 4(b), ten sublicenses are allocated. Since they are close to each other, we cannot really tell the exclusive boundary of every allocated sublicenses and cannot guarantee QoS at the boundary. One the right side, the top two contours share one boundary tile. More simulation results will be presented in Section IV.

\section{Allocation}

An interference graph $G$ could be constructed upon the interference conditions determined as above in Fig. 3. Let vertices denote the base stations and edges denote the interference condition between them. If the base station $i$ 's contour (interior tile) overlaps base station $j$ 's contour, we say there is interference between them (we do not count the overlapping boundary tiles as interfering tiles). Then the next step is to finding the independent vertices from the graph, which is modeled as graph coloring problem and proved to be NP-hard. Greedy algorithms are used for winner determination and the detailed further work can refer to [10] [13]. The allocation $a_{i}$ is determined in this step.

\section{Simulation Result}

In this section, we evaluate the performance of the proposed scheme, such as how the grid resolution and boundary sensitivity affect the allocation result and profit.

\section{A. Methodology}

We assume that $N=20$ base stations are uniformly distributed in a $2 \mathrm{~km} \times 2 \mathrm{~km}$ census tract. To make this model more practical we further let the $-96 \mathrm{dBm}$ contour of base station's radius be 200 meters. We run the simulation for 1000 times to mitigate the impact of randomness. In the simulation, the profit under different resolutions are compared and analyzed.

\section{B. Resolution and Interference Graph}

We first demonstrate how the resolution affects the Interference Graph, as shown in Fig. 3. An interference graph is widely used to assign spectrum to mutual interfering users. The nodes in the interference graph denote the users while the edges denote the interference between them. In Fig. 3, the graph differently formed as the resolution $\rho$ varies from $1 / 0.05^{2}$ to $1 / 0.4^{2}$. The interference graph is used for winner determination, so it directly results in a different allocation, as shown in Fig. 4. The color scale indicates the number of shared SPAL users. Particularly, when the tile's color value is -1 , it is a whole tile and not shared.

When the resolution is high, the region is fine gridded and the interference graph is more complex than that under a low resolution. Since the range of the base station is $0.2 \mathrm{~km}$, the minimum interference-free distance between two base stations is $0.4 \mathrm{~km}$. When the distance of the two neighboring sensors is larger than $0.4 \mathrm{~km}$, the sensing network cannot measure the area well.
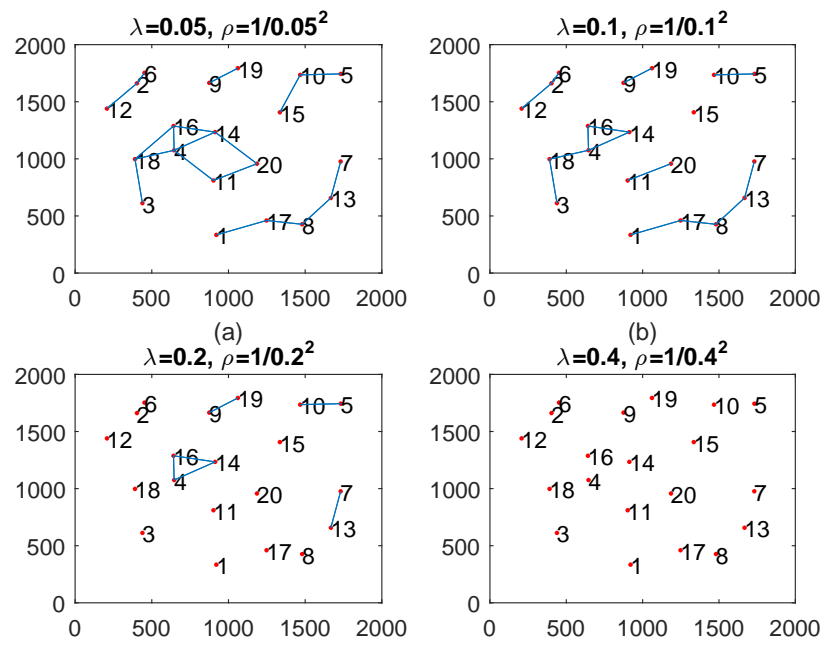

(c)

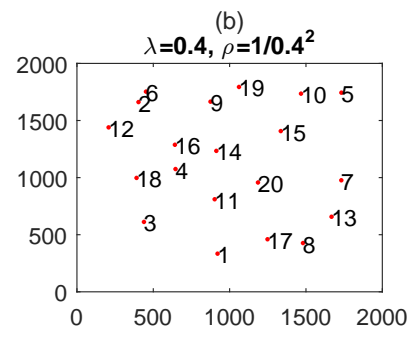

(d)

Fig. 3. Area $2 \mathrm{~km} \times 2 \mathrm{~km}$ : when the grid resolution varies from high to low, the interference graph becomes less complex.

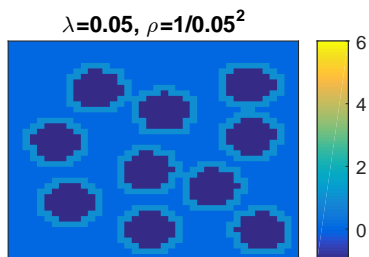

(a)

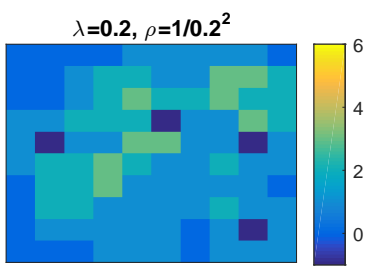

(c)

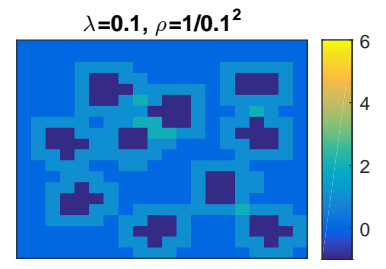

(b)

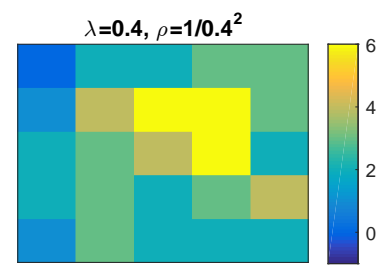

(d)
Fig. 4. Sublicenses allocation. When the grid resolution varies from high to low, the protection zones cannot be measured precisely. The tile's color scale indicates the number of shared SPAL users in that tile.

\section{Boundary Sensitivity}

The boundary sensitivity will be used to judge the tolerance of the shared users, particularly in a competitive situation. As illustrated in Fig. 5, if $\beta$ is set as zero, the contour boundary tiles cannot shared by anyone else, accordingly the boundary tiles will be ignored then profitable area is less. However, when $\beta$ is greater than zero, the total allocated area will increase then drop. Because the boundary area is usually partially covered by the half tiles, if the boundary area is shared by a limited number of users (the shared users only pay less), the total allocated area will increase with a little lower QoS. However, when the sensors are deployed every more than $0.125 \mathrm{~km}$, the grid is too coarse to distinguish the protection zones, then the profitable allocated area falls down. Because the severely interfering boundary tiles are ignored, and the estimation of protection zones is no longer precise. 


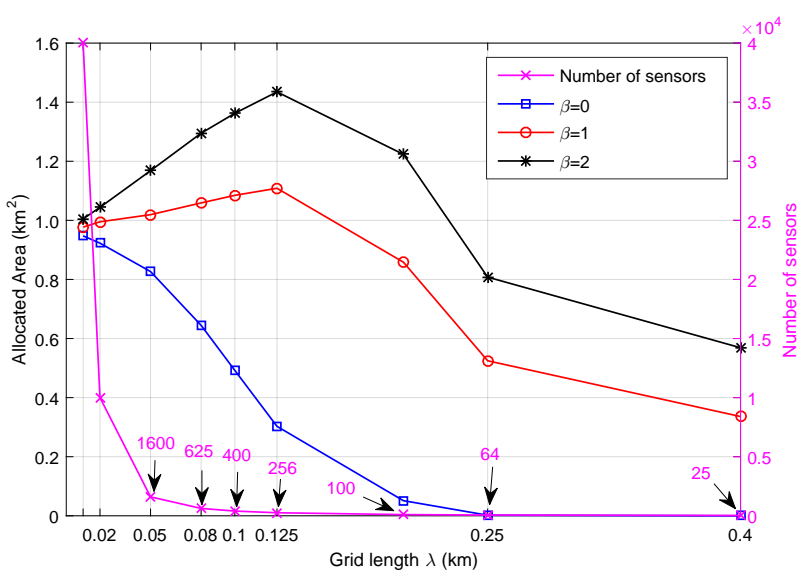

Fig. 5. The number of the sensors will exponentially decrease along with the grid length. When the boundary sensitivity is set greater than zero, the total allocated area is optimized at $\lambda=0.125$.

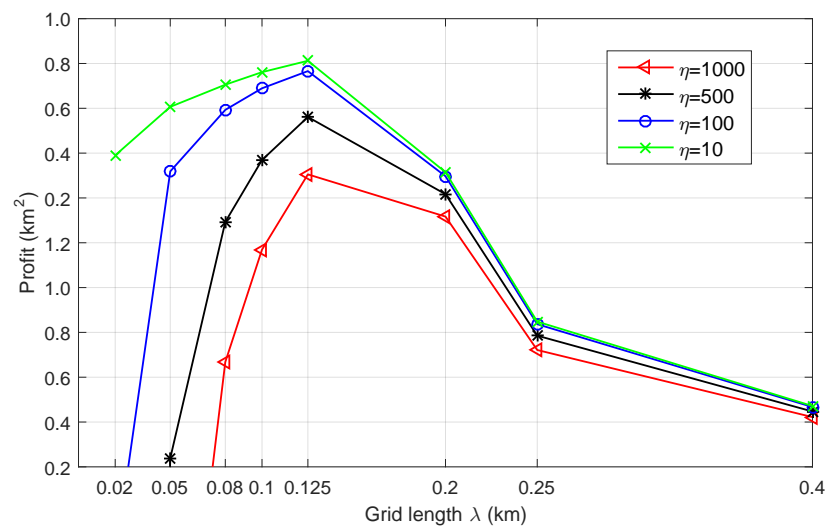

Fig. 6. With the decrement of the grid resolution, no matter how the cost of sensor changes, the profit reaches the peak at $\lambda=0.125$.

\section{Resolution and Profit}

As illustrated in Fig. 6, the grid length $\lambda$ varies from 0.01 $\mathrm{km}$ to $0.4 \mathrm{~km}$, and $\beta=2$. The valuation over per unit space is normalized as $v_{i}(x, y)=1$, while the cost $\eta$ of the each sensor is set as $\{10,100,500,1000\}$, compared to $v_{i}$. The profit first increases from extremely low to the peak until $\lambda=0.125 \mathrm{~km}$, then drops. This is because, when the resolution is high $(\lambda$ is small), even though the contours can be precisely measured, the cost of all the sensors is so expensive. When the resolution is low, the size of tile is large, then the estimation of contour area will be less precise. Accordingly, the cost is lower and the profit falls down. When the sensor is deployed every 0.125 $\mathrm{km}$, we can achieve the highest profit.

\section{CONCLUSION}

Spectrum sublicensing for temporary use is a flexible and feasible solution for spectrum sharing between multiple operators, particularly on the platform of SAS and LSA. In this paper, we propose a sublicensing scheme to enable the spectrum trading in a secondary market, hence the spectrum efficiency can be improved as driven by business. Operation of small cell networks only based on dedicated licenses may not feasible as more spectrum is needed, flexible spectrum use and short-term spectrum access is a way forward for profitable sharing in the coming decades. The principle of allocation spectrum to mobile network operators based on a dedicated and exclusive license will persist, as a method to ensure contour coverage and QoS. The proposed scheme can provide subsequent auction or other economic models with a reasonable interference graph. Since spectrum sharing in SAS has not yet been fully studied, this paper demonstrates how to open a secondary spectrum market and assign available sublicenses to SPAL users. Based on the analysis of the simulation results, the importance of the boundary sensitivity should be adjusted by the density of interested SPAL users and the resolution also be optimized to maximize the profit.

\section{ACKNOWLEDGMENT}

This work has been supported in part by Intel's University Research Office.

\section{REFERENCES}

[1] M. Abitbol and P. J. Muller, "Licensed Shared Access; an innovation in european radio spectrum policy," in Dynamic Spectrum Access Networks (DYSPAN), 2014 IEEE International Symposium on, April 2014, pp. 259-262.

[2] Radio spectrum policy group (RSPG), "Report on collective use of spectrum and other spectrum sharing approaches," RSPG11-392 Final, 2011.

[3] The White House, "Realizing the full potential of government-held spectrum to spur economic growth," Executive Office of the President, 2012.

[4] Federal Communications Commission, "Order on reconsideration and second report and order," vol. FCC-16-55, April 2016.

[5] T. Irnich, J. Kronander, Y. Selen, and G. Li, "Spectrum sharing scenarios and resulting technical requirements for $5 \mathrm{~g}$ systems," in Personal, Indoor and Mobile Radio Communications (PIMRC Workshops), 2013 IEEE 24th International Symposium on, Sept 2013, pp. 127-132.

[6] A. Weber and O. Stanze, "Scheduling strategies for hetnets using eicic," in Communications (ICC), 2012 IEEE International Conference on, June 2012, pp. 6787-6791.

[7] B. Singh, K. Koufos, O. Tirkkonen, and R. Berry, "Co-primary interoperator spectrum sharing over a limited spectrum pool using repeated games," in 2015 IEEE International Conference on Communications (ICC), June 2015, pp. 1494-1499.

[8] A. K. Sadek, W. Zhang, and S. J. Shellhammer, "Listen-before-talk versus treating interference as noise for spectrum sharing," in New Frontiers in Dynamic Spectrum Access Networks, 2008. DySPAN 2008. 3rd IEEE Symposium on, Oct 2008, pp. 1-6.

[9] H.-S. Jo, Y. J. Sang, P. Xia, and J. Andrews, "Heterogeneous cellular networks with flexible cell association: A comprehensive downlink sin analysis," Wireless Communications, IEEE Transactions on, vol. 11, no. 10, pp. 3484-3495, October 2012.

[10] H. Wang, E. Dutkiewicz, G. Fang, and M. D. Mueck, "Framework of joint auction and mixed graph for licensed shared access systems," in $D y$ namic Spectrum Access Networks (DySPAN), 2015 IEEE International Symposium on, Sept 2015, pp. 154-163.

[11] W. Dong, S. Rallapalli, L. Qiu, K. K. Ramakrishnan, and Y. Zhang, "Double auctions for dynamic spectrum allocation," in IEEE INFOCOM 2014 - IEEE Conference on Computer Communications, April 2014, pp. 709-717.

[12] H. ElSawy, E. Hossain, and M. Haenggi, "Stochastic geometry for modeling, analysis, and design of multi-tier and cognitive cellular wireless networks: A survey," Communications Surveys Tutorials, IEEE, vol. 15, no. 3, pp. 996-1019, Third 2013.

[13] T. Jing, C. Zhao, X. Xing, Y. Huo, W. Li, and X. Cheng, "A multi-unit truthful double auction framework for secondary market," in Communications (ICC), 2013 IEEE International Conference on, June 2013, pp. 2817-2822. 